\title{
Efficient General-Adversary Multi-Party Computation
}

\author{
Martin Hirt and Daniel Tschudi ${ }^{\star}$ \\ ETH Zurich \\ \{hirt, tschudid\}ainf.ethz.ch
}

\begin{abstract}
Secure multi-party computation (MPC) allows a set $\mathcal{P}$ of $n$ players to evaluate a function $f$ in presence of an adversary who corrupts a subset of the players. In this paper we consider active, general adversaries, characterized by a so-called adversary structure $\mathcal{Z}$ which enumerates all possible subsets of corrupted players. In particular for small sets of players general adversaries better capture real-world requirements than classical threshold adversaries.

Protocols for general adversaries are "efficient" in the sense that they require $|\mathcal{Z}|^{\mathcal{O}(1)}$ bits of communication. However, as $|\mathcal{Z}|$ is usually very large (even exponential in $n$ ), the exact exponent is very relevant. In the setting with perfect security, the most efficient protocol known to date communicates $\mathcal{O}\left(|\mathcal{Z}|^{3}\right)$ bits; we present a protocol for this setting which communicates $\mathcal{O}\left(|\mathcal{Z}|^{2}\right)$ bits. In the setting with statistical security, $\mathcal{O}\left(|\mathcal{Z}|^{3}\right)$ bits of communication is needed in general (whereas for a very restricted subclass of adversary structures, a protocol with communication $\mathcal{O}\left(|\mathcal{Z}|^{2}\right)$ bits is known); we present a protocol for this setting (without limitations) which communicates $\mathcal{O}\left(|\mathcal{Z}|^{1}\right)$ bits.
\end{abstract}

Keywords: Secure Multiparty Computation, General Adversaries, Efficiency.

\section{Introduction}

Secure Multi-Party Computation. Secure Multi-Party Computation (MPC) allows a set $\mathcal{P}$ of $n$ players to securely evaluate a function $f$ even when a subset of the players is corrupted by a central adversary. MPC was introduced by Yao [Yao82]. A first solution (with computational security) was given by Goldreich, Micali, and Wigderson [GMW87]. Later solutions [BGW88, CCD88, RB89] provide statistical and even perfect security. All these protocols consider threshold adversaries (characterized by an upper bound $t$ on the number of corrupted parties). This was generalized in [HM00] by considering so-called general adversaries, characterized by an adversary structure $\mathcal{Z}=\left\{Z_{1}, \ldots, Z_{\ell}\right\}$, which enumerates all possible subsets of corrupted players.

In the setting with perfect active security, MPC is achievable if and only if $t<\frac{n}{3}$, respectively $\mathcal{Q}^{3}(\mathcal{P}, \mathcal{Z})$ (the union of no three sets in $\mathcal{Z}$ covers $\mathcal{P}$ ). In the setting with statistical or cryptographic active security, MPC is achievable if and only if $t<\frac{n}{2}$, respectively $\mathcal{Q}^{2}(\mathcal{P}, \mathcal{Z})$ (the union of no two sets in $\mathcal{Z}$ covers $\mathcal{P}$ ).

\footnotetext{
* Research supported in part by the Swiss National Science Foundation (SNF), project no. 200020-132794. 
Threshold vs. General Adversaries. Clearly, general adversaries are more flexible, which is relevant in particular when the set of players is not very large. However, general-adversary protocols are typically by orders of magnitude less efficient than threshold protocols; more specifically, threshold protocols usually communicate $\operatorname{Poly}(n)$ bits per multiplication, whereas general-adversary protocols require $\operatorname{Poly}(|Z|)$ bits. As typically $|\mathcal{Z}|$ is exponential in $n$, this is a huge drawback. However, in some scenarios (e.g. with very different types of players), threshold protocols are not applicable, and general-adversary protocols must be used. In these settings, the concrete communication complexity of the general-adversary protocol is highly relevant: For example for $n=25,|\mathcal{Z}|$ is expected to be around one million, and a protocol communicating $|\mathcal{Z}| \cdot \operatorname{Poly}(n)$ might be acceptable, whereas a protocol communicating $|\mathcal{Z}|^{3} \cdot \operatorname{Poly}(n)$ might be useless.

Contributions. In the statistically-secure model, one can tolerate at most adversary structures satisfying $\mathcal{Q}^{2}(\mathcal{P}, \mathcal{Z})$. The most efficient protocol known to date, which is also optimal in terms of resilience, requires $|\mathcal{Z}|^{3} \cdot \operatorname{Poly}(n, \kappa)$ bits of communication (where $\kappa$ is the security parameter) [Mau02, HMZ08]. There exists a protocol with communication complexity of $|\mathcal{Z}|^{2} \cdot \operatorname{Poly}(n, \kappa)$ [PSR03]. But this results is non-optimal in terms of resilience, as it tolerates only adversaries satisfying $\mathcal{Q}^{3}$.

Using a new approach for multiplication, we construct a protocol communicating $|\mathcal{Z}| \cdot \operatorname{Poly}(n, \kappa)$ bits and tolerating $\mathcal{Q}^{2}$ adversary structures. This protocol is optimal both in terms of overall efficiency and resilience. We stress that even with cryptographic security, $\mathcal{Q}^{2}$ is necessary and complexity linear in $|\mathcal{Z}|$ is required at least with respect to the computation (see [Hir01]).

Furthermore, we present a perfectly secure protocol (with no error probability) with communication complexity of $|\mathcal{Z}|^{2}$. $\operatorname{Poly}(n)$. It is optimal in terms of resilience $\left(\mathcal{Q}^{3}\right)$ and also the most efficient protocol up to date in the model with perfect security.

Table 1. Communication complexity of different protocols

\begin{tabular}{|l|c|c|c|}
\hline Setting & Cond. & Bits / Mult. & Reference \\
\hline passive perfect & $\mathcal{Q}^{2}$ & $|\mathcal{Z}| \cdot \operatorname{Poly}(n)$ & {$[$ Mau02] } \\
\hline active perfect & $\mathcal{Q}^{3}$ & $|\mathcal{Z}|^{3} \cdot \operatorname{Poly}(n)$ & {$[$ Mau02] } \\
active perfect & $\mathcal{Q}^{3}$ & $|\mathcal{Z}|^{2} \cdot \operatorname{Poly}(n)$ & our result \\
\hline active unconditional & $\mathcal{Q}^{2}$ & $|\mathcal{Z}|^{3} \cdot \operatorname{Poly}(n, \kappa)$ & {$[$ Mau02]/[HMZ08] } \\
active unconditional & $\mathcal{Q}^{3}$ & $|\mathcal{Z}|^{2} \cdot \operatorname{Poly}(n, \kappa)$ & {$[$ [PSR03] } \\
active unconditional & $\mathcal{Q}^{2}$ & $|\mathcal{Z}| \cdot \operatorname{Poly}(n, \kappa)$ & our result \\
\hline
\end{tabular}

\section{Preliminaries}

Players and Computation. Let $\mathcal{P}=\left\{P_{1}, \ldots, P_{n}\right\}$ be a set of $n$ players. The players in $\mathcal{P}$ want to compute a function $f$ over some finite field $\mathbb{F}$. The function is specified by a circuit $\mathcal{C}$ consisting of input, output, random, addition, and multiplication gates. 
In an ideal world, a trusted party does all the computation. In the real world, players are connected by a complete network of secure (private and authentic) synchronous channels. There exist authenticated broadcast channels (they can be simulated by the players, see e.g. [FM98] or [PW96] ). In order to compute the function $f$, the players simulate the trusted party by using some MPC-protocol $\Pi$.

Adversary and Adversary Structure. Dishonesty is modeled in terms of a central adversary $\mathcal{A}$ who corrupts players. During the execution of the protocol the adversary can access the internal state of corrupted players and make them deviate from the protocol at will. We allow that the adversary is computationally unbounded. Before the execution of the protocol the adversary has to specify the players he wants to corrupt. His choice is limited by means of an adversary structure $\mathcal{Z}=\left\{Z_{1}, \ldots, Z_{\ell}\right\} \subseteq 2^{\mathcal{P}}$, i.e. all corrupted players must be part of an adversary set in $\mathcal{Z}$. We denote the chosen set by $Z^{*}$. Note that $Z^{*}$ is not known to the honest players and is solely used for ease of notation. We say that $\mathcal{Z}$ satisfies the $\mathcal{Q}^{k}(\mathcal{P}, \mathcal{Z})$ property if $\mathcal{P} \nsubseteq Z_{1} \cup \cdots \cup Z_{k} \quad \forall Z_{1}, \ldots, Z_{k} \in \mathcal{Z}$.

Security. We say a protocol is $\mathcal{Z}$-secure if anything the adversary achieves during the execution of the protocol can be achieved in the ideal world as well. More precisely, for every adversary in the real world there exists an adversary in the ideal world such that both the information the adversary gets and the output of honest players are statistically indistinguishable for perfect security respectively statistically close for unconditional security. The main result from [HM97] states that $\mathcal{Q}^{3}(\mathcal{P}, \mathcal{Z})$ resp. $\mathcal{Q}^{2}(\mathcal{P}, \mathcal{Z})$ are the necessary and sufficient conditions for the existence of perfectly resp. unconditionally $\mathcal{Z}$-secure protocols considering active adversaries. For simplicity we assume that all messages sent during the execution of $\Pi$ are from the right domain. If a player receives a message where this is not the case, he replaces it with an arbitrary element from the right domain. If a player receives an unexpected message, he ignores it.

\section{Perfect Protocol}

In this section we present a perfectly $\mathcal{Z}$-secure protocol for an arbitrary adversary structure $\mathcal{Z}$ satisfying the $\mathcal{Q}^{3}$ property. The communication complexity of the protocol is quadratic in $|\mathcal{Z}|$. The efficiency gain is due to an improved multiplication protocol. The sharing is (up to presentation) the same as in [Mau02].

\subsection{Secret Sharing}

Secret sharing allows a player to distribute a secret value among the player set, such that only qualified subsets of players are able reconstruct it. The secret sharing used for our protocol is based on the one from [Mau02] / $\left.\mathrm{BFH}^{+} 08\right]$. It is characterized by a sharing specification $\mathbb{S}=\left(S_{1}, \ldots, S_{h}\right)$, which is a tuple of subsets of $\mathcal{P}$.

Definition 1. A value $s$ is shared with respect to sharing specification $\mathbb{S}=\left(S_{1}, \ldots, S_{h}\right)$ if the following holds: 
a) There exist shares $s_{1}, \ldots, s_{h}$ such that $s=\sum_{q=1}^{h} s_{q}$

b) Each $s_{q}$ is known to every (honest) player in $S_{q}$

We denote the sharing of a value $s$ by $[s]$ and use $[s]_{q}$ as notation for $s_{q}$, the $q$-th share. A sharing specification $\mathbb{S}=\left(S_{1}, \ldots, S_{h}\right)$ is called $\mathcal{Z}$-private if for every $Z \in \mathcal{Z}$ there is an $S \in \mathbb{S}$ such that $Z \cap S=\emptyset$. A sharing specification $\mathbb{S}=\left(S_{1}, \ldots, S_{h}\right)$ and an adversary structure $\mathcal{Z}$ satisfy $\mathcal{Q}^{k}(\mathbb{S}, \mathcal{Z})$ if $S \nsubseteq Z_{1} \cup \cdots \cup Z_{k} \quad \forall Z_{1}, \ldots, Z_{k} \in$ $\mathcal{Z} S \in \mathbb{S}$. If $\mathbb{S}$ is $\mathcal{Z}$-private, a sharing $[s]$ does not leak information to the adversary, as all shares known by the adversary are statistically independent of $s$. The players can compute a sharing of any linear combination of shared values (with respect to a sharing specification $\mathbb{S}$ ) by locally computing the linear combination of their shares. This property is called the linearity of the sharing. The following protocol Share allows a dealer $P_{D}$ to correctly share value $s$ among the players in $\mathcal{P}$.

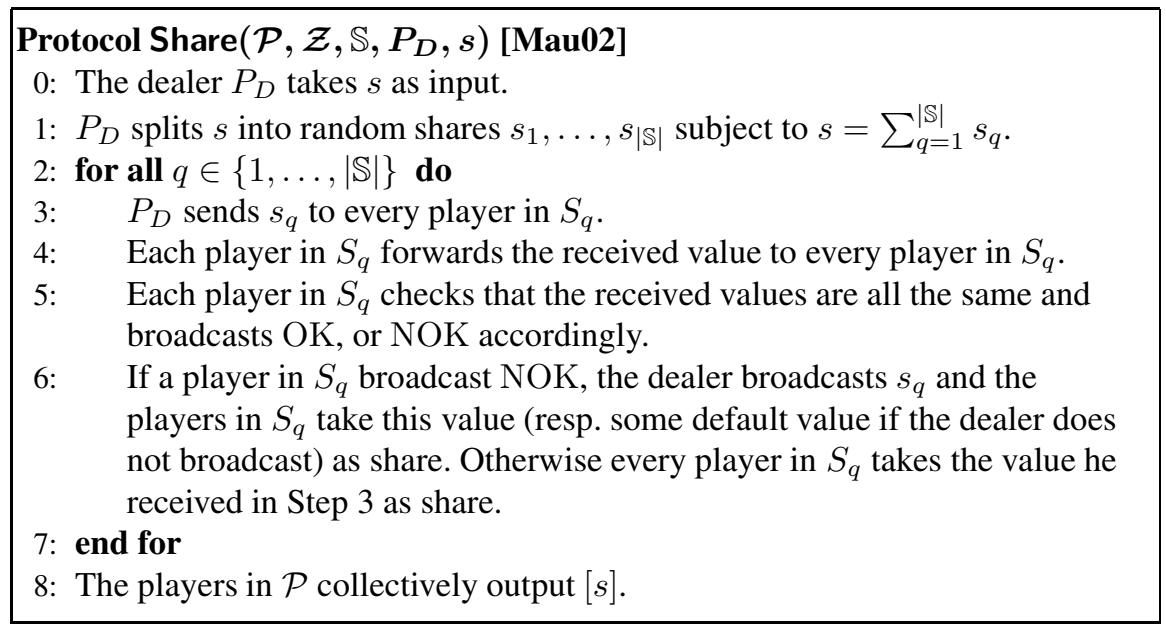

Lemma 1. For any adversary structure $\mathcal{Z}$ the protocol $\operatorname{Share}\left(\mathcal{P}, \mathcal{Z}, \mathbb{S}, P_{D}, s\right)$ securely computes a sharing $\left[s^{\prime}\right]$. For honest $P_{D}$ it holds that $s^{\prime}=s$. The protocol communicates at most $|\mathbb{S}|\left(n^{2}+n\right) \log |\mathbb{F}|$ bits and broadcasts at most $|\mathbb{S}|(\log |\mathbb{F}|+n)$ bits.

Proof. Correctness: For each $s_{q}$ either all the honest players in $S_{q}$ hold the same value after Step 3, or one of them complains and they receive a consistent value in Step 6 . Hence the protocol outputs a (consistent) sharing $\left[s^{\prime}\right]$. If the dealer is honest he is able to ensure in Steps 3 and 6 that the honest players use the intended value for $s_{q}$ such that $s=s^{\prime}$. Privacy: Let the dealer be honest, as otherwise secrecy is trivially fulfilled. All a player learns beyond his designated output are values broadcast in Step 6 However this does not violate secrecy as these values are already known to the adversary (from Step 3). Complexity: For each share at most $n+n^{2}$ values are sent and at most $n+\log |\mathbb{F}|$ bits broadcast.

For publicly known value $s$ the players can invoke DefaultShare to get a sharing $[s]$ without having to communicate. 
Protocol DefaultShare $(\mathcal{P}, \mathcal{Z}, \mathbb{S}, s)$

0 : Every player takes $s$ as input.

1: The share $s_{1}$ is set to $s$ and all other shares are set to 0 .

2: The players in $\mathcal{P}$ collectively output $[s]$.

Lemma 2. Default $\operatorname{Share}(\mathcal{P}, \mathcal{Z}, \mathbb{S}, s)$ securely computes a sharing $[s]$ where $s$ is a publicly known value. The protocol does not communicate.

Proof. Correctness: In Step 1 every honest player in $S_{q}$ takes the same value for share $s_{q}$. As the sum of all shares is $s$, the protocol outputs a consistent sharing $[s]$. Privacy: During the protocol no communication occurs, hence the adversary does not obtain new information.

The protocol ReconstructShare allows the reconstruction of a share $[s]_{q}$ to the players in some set $R$. This implies that the players can reconstruct a shared value $[s]$ by invoking ReconstructShare for each share.

Protocol ReconstructShare $\left(\mathcal{P}, \mathcal{Z}, \mathbb{S},[s]_{q}, R\right)$

0: The players in $S_{q}$ take the share $[s]_{q}$ as input.

1: Every player $P_{i}$ in $S_{q}$ sends $[s]_{q}$ to every player in $R$.

2: For each player $P_{j} \in R$ let $v_{j, i}$ be the value received from $P_{i}$. Then $P_{j}$ outputs some value $v_{j}$ such that there exists a $Z \in \mathcal{Z}$ with $v_{j, i}=v_{j}$ for all $P_{i} \in S_{q} \backslash Z$.

Lemma 3. If $S_{q}$ and $\mathcal{Z}$ satisfy $\mathcal{Q}^{2}\left(S_{q}, \mathcal{Z}\right)$, the protocol ReconstructShare securely reconstructs the share $[s]_{q}$ to the players in $R$, such that every (honest) player outputs $[s]_{q}$. The protocol communicates at most $n^{2} \log |\mathbb{F}|$ bits.

Proof. Correctness: In Step 1 all honest player will send the same value $[s]_{q}$, which is a suitable choice for $v_{j}$ for an (honest) player $P_{j} \in R$ in Step 2] For the sake of contradiction suppose there exist two values $v_{1} \neq v_{2}$ with corresponding $Z_{1}, Z_{2} \in \mathcal{Z}$ such that the condition of Step 2 holds for both of them. Hence $\left(S_{q} \backslash Z_{1}\right) \cap\left(S_{q} \backslash Z_{2}\right)=\emptyset$ and thus $S_{q} \subseteq Z_{1} \cup Z_{2}$ which contradicts $\mathcal{Q}^{2}\left(S_{q}, \mathcal{Z}\right)$. Therefore every honest players outputs the value $[s]_{q}$. Privacy: The adversary learns at most $[s]_{q}$ (if a malicious player is part of $R$ ). Complexity: Each player in $S_{q}$ sends his value to at most $n$ players.

$\operatorname{Protocol} \operatorname{Reconstruct}(\mathcal{P}, \mathcal{Z}, \mathbb{S},[s], R)[\overline{M a u 02}]$

0 : The players in $\mathcal{P}$ take collectively $[s]$ as input.

1: $\forall q \in\{1, \ldots,|\mathbb{S}|\}$ protocol ReconstructShare $\left(\mathcal{P}, \mathcal{Z}, \mathbb{S},[s]_{q}, R\right)$ is invoked.

2: The players in $R$ locally sum up the obtained shares and output the sum $s$.

Lemma 4. If $\mathbb{S}$ and $\mathcal{Z}$ satisfy $\mathcal{Q}^{2}(\mathbb{S}, \mathcal{Z})$ and $[s]$ is a sharing of the value $s$, then $\operatorname{Reconstruct}(\mathcal{P}, \mathcal{Z}, \mathbb{S},[s], R)$ securely reconstructs $s$ to the players in $R$. The protocol communicates at most $|\mathbb{S}| n^{2} \log |\mathbb{F}|$ bits.

Proof. Correctness and privacy follow directly from Lemma 3. As ReconstructShare is invoked $|\mathbb{S}|$ times the complexity follows as well. 


\subsection{Multiplication}

We present a protocol for the perfectly-secure computation of the (shared) product of two shared values $[a]$ and $[b]$ (with respect to a sharing specification $\mathbb{S}$ ). Along the lines of [Mau02] the fundamental idea of multiplication is to assign each local product $a_{p} b_{q}$ to a player in $S_{p} \cap S_{q}$, who computes and shares his designated products. The sum of all these sharings is a sharing of $a b$ as long as no player actively cheated. So each player is mapped to a collection of local products, formalized by a function $I$ : $[n] \rightarrow 2^{\{(p, q)|1 \leq p, q \leq| \mathcal{Z} \mid\}}$ with the constraint that $\forall(p, q) \exists ! i$ such that $(p, q) \in I(i)$. W.1.o.g let $I(i):=\left\{(p, q) \mid P_{i}=\min _{P}\left\{P \in S_{p} \cap S_{q}\right\}\right\}$. We first show an optimistic multiplication protocol which takes an additional parameter $Z$ and computes the correct product if the actual adversary set $Z^{*}$ is a subset of $Z$. In this protocol local products are assigned to players in $\mathcal{P} \backslash Z$ only. Clearly this is possible if and only if for each local product a player in $\mathcal{P} \backslash Z$ holds both involved shares, i.e. $\forall S_{p}, S_{q} \in \mathbb{S}: S_{p} \cap S_{q} \backslash Z \neq \emptyset$. So for each $Z \in \mathcal{Z}$ let $I_{Z}$ be a mapping as above with the additional constraint that $\forall P_{i} \in Z I_{Z}(i)=\emptyset$. Without loss of generality, let $I_{Z}(i):=\left\{(p, q) \mid P_{i}=\min _{P}\{P \in\right.$ $\left.\left.S_{p} \cap S_{q} \backslash Z\right\}\right\}$.

Protocol OptimisticMult $(\mathcal{P}, \mathcal{Z}, \mathbb{S},[a],[b], Z)$

0 : The players in $\mathcal{P}$ take collectively $[a],[b]$ and $Z$ as input.

1:

a) Each player $P_{i} \in \mathcal{P} \backslash Z$ (locally) computes his designated products and shares the sum $c_{i}=\sum_{(p, q) \in I_{Z}(i)} a_{p} b_{q}$.

b) For each $P_{i} \in Z$ DefaultShare $(\mathcal{P}, \mathcal{Z}, \mathbb{S}, 0)$ is invoked to share $c_{i}=0$.

2: The players collectively output $\left(\left[c_{1}\right], \ldots,\left[c_{n}\right]\right)$ and $[c]=\sum_{i=1}^{n}\left[c_{i}\right]$.

Lemma 5. Let $Z \subseteq \mathcal{P}$ such that $\forall S_{p}, S_{q} \in \mathbb{S}: S_{p} \cap S_{q} \backslash Z \neq \emptyset$. Then the protocol OptimisticMult securely computes sharings $[c],\left(\left[c_{1}\right], \ldots,\left[c_{n}\right]\right)$. If no player in $\mathcal{P} \backslash Z$ actively cheats (in particular, if $Z^{*} \subseteq Z$ ), then $\forall i c_{i}=\sum_{(p, q) \in I_{Z}(i)} a_{p} b_{q}$ and $c=$ ab. The protocol communicates at most $\mathcal{O}\left(|\mathbb{S}| n^{3} \log |\mathbb{F}|\right)$ bits and broadcasts at most $\mathcal{O}\left(|\mathbb{S}|\left(n \log |\mathbb{F}|+n^{2}\right)\right)$ bits.

Proof. Correctness: The properties of the sharing protocol guarantee that the outputs are valid sharings. If none of the players in $\mathcal{P} \backslash Z$ cheated actively, it holds for each $P_{i}$ that $c_{i}=\sum_{(p, q) \in I_{Z}(i)} a_{p} b_{q}$. The condition $\forall S_{p}, S_{q} \in \mathbb{S}: S_{p} \cap S_{q} \backslash Z \neq \emptyset$ guarantees that $a b=\sum_{i=1}^{n} \sum_{(p, q) \in I_{Z}(i)} a_{p} b_{q}$. Hence it follows that $c=a b$. Privacy / Complexity: Follow directly from Lemmas 1 and 2 .

As the players do not know the actual adversary set $Z^{*}$, they invoke OptimisticMult once for each set $Z \in \mathcal{Z}$ (Step 1 of the Multiplication protocol). This guarantees that at least one of the resulting sharings is correct. By comparing them the players can determine whether cheating occurred (Step 2] of the Multiplication protocol). If all sharings are equal, no cheating occurred and any of the sharings can serve as sharing of the product. Otherwise at least one player cheated. In this case the (honest) players can identify him and remove all sharings where he was involved in computation, 
as these sharings are potentially tampered (Step 3 of the Multiplication protocol). This checking and removing is repeated until all remaining sharing are equal (and hence correct). As the identification of cheaters does not reveal any information to the adversary, Multiplication allows the secure computation of the product of two shared secret values.

\section{Protocol Multiplication $(\mathcal{P}, \mathcal{Z}, \mathbb{S},[a],[b])$}

0: Set $M=\emptyset$.

1: Invoke OptimisticMult $(\mathcal{P}, \mathcal{Z}, \mathbb{S},[a],[b], Z)$ to compute $\left(\left[c_{1}^{(Z)}\right], \ldots,\left[c_{n}^{(Z)}\right]\right)$ and $\left[c^{(Z)}\right]$ for each $Z \in \mathcal{Z}$.

2: Set $\mathcal{Z}_{M}:=\{Z \in \mathcal{Z} \mid M \subseteq Z\}$, fix some $\widetilde{Z} \in \mathcal{Z}_{M}$ and reconstruct the differences $\left[c^{(\widetilde{Z})}\right]-\left[c^{(Z)}\right] \forall Z \in \mathcal{Z}_{M}$.

3: If all differences are zero, output $\left[c^{(\widetilde{Z})}\right]$ as sharing of the product.

Otherwise let $\left(\left[d_{1}\right], \ldots,\left[d_{n}\right]\right):=\left(\left[c_{1}^{(\widetilde{Z})}\right], \ldots,\left[c_{n}^{(\widetilde{Z})}\right]\right),\left(\left[e_{1}\right], \ldots,\left[e_{n}\right]\right):=$ $\left(\left[c_{1}^{(Z)}\right], \ldots,\left[c_{n}^{(Z)}\right]\right), D:=I_{\widetilde{Z}}$ and $E:=I_{Z}$, where $\left[c^{(\widetilde{Z})}\right]-\left[c^{(Z)}\right] \neq 0$.

a) Each $P_{i}$ shares the $2 n$ values $d_{i, j}=\sum_{(p, q) \in D(i) \cap E(j)} a_{p} b_{q}$ and $e_{i, j}=$ $\sum_{(p, q) \in E(i) \cap D(j)} a_{p} b_{q}$

b) For each player $P_{i}$ reconstruct the differences $\left[d_{i}\right]-\sum_{j=1}^{n}\left[d_{i, j}\right]$ and $\left[e_{i}\right]-$ $\sum_{j=1}^{n}\left[e_{i, j}\right]$. If one of them is non-zero set $M \leftarrow M \cup\left\{P_{i}\right\}$ and continue at Step 2.

c) For each (ordered) pair $\left(P_{i}, P_{j}\right)$ of players reconstruct the difference $\left[d_{i, j}\right]-\left[e_{j, i}\right]$. If it is non-zero, reconstruct $\left[d_{i, j}\right],\left[e_{j, i}\right]$ and all shares $\left\{a_{p}, b_{q} \mid(p, q) \in D(i) \cap E(j)\right\}$ to find the cheater $P \in\left\{P_{i}, P_{j}\right\}$. Set $M \leftarrow M \cup\{P\}$ and continue at Step 2 ]

Lemma 6. If $\mathbb{S}$ and $\mathcal{Z}$ satisfy $\mathcal{Q}^{2}(\mathbb{S}, \mathcal{Z})$ the protocol Multiplication yields a sharing $[c]=[a b]$. No information is leaked to the adversary. Multiplication communicates at most $\mathcal{O}\left(|\mathbb{S}||\mathcal{Z}| n^{3} \log |\mathbb{F}|+|\mathbb{S}| n^{5} \log |\mathbb{F}|\right)$ bits and broadcasts at most $\mathcal{O}(|\mathbb{S}||\mathcal{Z}|(n$ $\left.\left.\log |\mathbb{F}|+n^{2}\right)+|\mathbb{S}|\left(n^{3} \log |\mathbb{F}|+n^{4}\right)\right)$ bits.

Proof. Correctness: By invoking OptimisticMult for each $Z \in \mathcal{Z}$ it holds for $Z^{*}$ that $\left[c^{\left(Z^{*}\right)}\right]=[a b]$ (due to $\mathcal{Q}^{2}(\mathbb{S}, \mathcal{Z}) \forall S_{p}, S_{q} \in \mathbb{S}: S_{p} \cap S_{q} \backslash Z \neq \emptyset$ holds). If for every $Z \in \mathcal{Z}_{M}$ the difference in Step 2 is zero, then $\left[c^{(Z)}\right]=[a b] \forall Z \in \mathcal{Z}_{M}(M=\emptyset$ at the beginning). Hence the protocol terminates successfully outputting a sharing of $a b$. Otherwise there exists $\left[c^{(\widetilde{Z})}\right]-\left[c^{(Z)}\right] \neq 0$ and thus $\sum_{i=1}^{n}\left[d_{i}\right] \neq \sum_{i=1}^{n}\left[e_{i}\right]$. In Step 3a) each player is supposed to share a partition of his shares. Hence one of the following cases must occur: There exists a player $P_{i}$ such that $\left[d_{i}\right] \neq \sum_{j=1}^{n}\left[d_{i, j}\right]$ or $\left[e_{i}\right] \neq \sum_{j=1}^{n}\left[e_{i, j}\right]$. Or there exists a pair of players $\left(P_{i}, P_{j}\right)$ such that $\left[d_{i, j}\right] \neq\left[e_{j, i}\right]$. In the first case $P_{i}$ will be detected as cheater in Step $3 \mathrm{~b}$ ). In the second case the cheater will be detected in Step 3r). In both cases $M \subseteq \mathcal{P}$ is strictly increased, hence the protocol will terminate after at most $n$ iterations. It holds that $M \subseteq Z^{*}$ and thus $Z^{*} \in$ $\mathcal{Z}_{M}$. Therefore the correct sharing $\left[c^{\left(Z^{*}\right)}\right]$ is always used in Step2] and the protocol will output the correct result. Privacy: By the properties of the sharing scheme and Lemma 5 the invocation of Share, Reconstruct, OptimisticMult does not violate privacy. The 
adversary learns the differences reconstructed in Steps 2 and 3 of Multiplication, which are all zero unless the adversary cheats. In case of cheating the reconstructed values depends solely on the inputs of the adversary and are thus already known to him, thus privacy is not violated. All values further reconstructed in Step 3k) are known to the adversary before, as either $P_{i}$ or $P_{j}$ is corrupted. Complexity: Follows from Lemmas 1 , 4 and 5 by counting the number of invocations of the corresponding sub-protocols.

\subsection{MPC Protocol}

Combining Share, Reconstruct, and Multiplication the players can securely compute a circuit $\mathcal{C}$ over $\mathbb{F}$, where all intermediate values are shared according to Definition 1 .

\section{$\operatorname{Protocol} \operatorname{MPC}(\mathcal{P}, \mathcal{Z}, \mathcal{C})$}

0: The players take $\mathbb{S}:=\{\mathcal{P} \backslash Z \mid Z \in \mathcal{Z}\}$ as sharing specification.

1: For every gate of $C$ being evaluated do the following:

- Input gate for $P_{D}$ : Share $\left(\mathcal{P}, \mathcal{Z}, \mathbb{S}, P_{D}, s\right)$ is invoked to share $s$, where $P_{D}$ is the input-giving player.

- Linear gate: The linear combination of the corresponding shares is computed locally using the linearity of the sharing.

- Random gate: Each player shares a random value. The sum of these values is used as output of the gate.

- Multiplication gate: Multiplication $(\mathcal{P}, \mathcal{Z}, \mathbb{S},[a],[b])$ is used to multiply $[a]$ and $[b]$.

- Output gate: The players invoke $\operatorname{Reconstruct}(\mathcal{P}, \mathcal{Z}, \mathbb{S},[s], R)$ to reconstruct the sharing $[s]$ to players in $R$.

Theorem 1. Let $\mathcal{P}$ be a set of n players, $\mathcal{C}$ a circuit over $\mathbb{F}$ and $\mathcal{Z}$ an adversary structure satisfying $\mathcal{Q}^{3}(\mathcal{P}, \mathcal{Z})$, then $\operatorname{MPC}(\mathcal{P}, \mathcal{Z}, \mathcal{C})$ perfectly $\mathcal{Z}$-securely evaluates $\mathcal{C}$. It communicates $|\mathcal{C}||\mathcal{Z}|^{2} \cdot \operatorname{Poly}(n, \log |\mathbb{F}|)$ bits.

Proof. It is easy to see that $\mathbb{S}:=\{\mathcal{P} \backslash Z \mid Z \in \mathcal{Z}\}$ is a sharing specification satisfying $\mathcal{Q}^{2}(\mathbb{S}, \mathcal{Z})$. Hence by the properties of the sharing scheme and Lemma 6 the statement follows. The protocol communicates $\mathcal{O}\left(|\mathcal{C}||\mathcal{Z}|^{2} n^{3} \log |\mathbb{F}|+|\mathcal{C}||\mathcal{Z}| n^{5} \log |\mathbb{F}|\right)$ bits and broadcasts $\mathcal{O}\left(|\mathcal{C}||\mathcal{Z}|^{2}\left(n \log |\mathbb{F}|+n^{2}\right)+|\mathcal{C}||\mathcal{Z}|\left(n^{3} \log |\mathbb{F}|+n^{4}\right)\right)$ bits. Broadcast can be simulated with the protocol in [FM98], which communicates $\operatorname{Poly}(n)$ bits in order to broadcast one bit. This yields the claimed communication complexity.

\section{Unconditional Protocol}

Our main result is an MPC protocol unconditionally $\mathcal{Z}$-secure for an $\mathcal{Q}^{2}$ adversary structure $\mathcal{Z}$. Its communication complexity is linear in $|\mathcal{Z}|$. This is the first protocol reaching the optimal lower bound of $\Omega(|\mathcal{Z}|)$ on the computational complexity (see Section 6). 


\subsection{Information Checking}

In the perfect model, $\mathcal{Q}^{3}$ enables the honest players to securely reconstruct shares, as it assures that every share is held by enough honest players. Here, $\mathcal{Q}^{2}$ only ensures that each share is held by at least one honest player. Correctness is achieved by the use of information checking, a technique that prevents (malicious) players from announcing wrong values (see [RB89, Bea91a, $\mathrm{CDD}^{+}$99, HMZ08]). The following informationchecking protocol is a slight variation of $\left[\mathrm{CDD}^{+99}\right]$. It is a three party protocol between a sender $P_{i}$, a recipient $P_{j}$ and a verifier $P_{k}$. The sender $P_{i}$ provides $P_{j}$ with some authentication tag and $P_{k}$ with some verification tag, such that $P_{j}$ later can prove the authenticity of a value $s$ to the verifier $P_{k}$. We assume that each pair $P_{i}, P_{k}$ of players knows a fixed secret value $\alpha_{i, k} \in \mathbb{F} \backslash\{0,1\}$.

Definition 2. A vector $(s, y, z, \alpha)$ is 1-consistent if there exists a polynomial $f$ of degree 1 over $\mathbb{F}$ such that $f(0)=s, f(1)=y, f(\alpha)=z$. We say a value $s$ is $\left(P_{i}, P_{j}, P_{k}\right)$ authenticated if $P_{j}$ knows $s$ and some authentication tag $y$ and $P_{k}$ knows a verification tag $z$ such that $\left(s, y, z, \alpha_{i, k}\right)$ is 1 -consistent. The vector $\left(y, z, \alpha_{i, k}\right)$ is denoted by $A_{i, j, k}(s)$.

Lemma 7. $A\left(P_{i}, P_{j}, P_{k}\right)$-authenticated value $s$ does not leak information to $P_{k}$.

Proof. The verification tag $z$ is statistically independent of the value $s$.

Lemma 8. Let $s$ be $\left(P_{i}, P_{j}, P_{k}\right)$-authenticated, i.e. $\left(s, y, z, \alpha_{i, k}\right)$ is 1-consistent. Then for $P_{j}$ being able to find an authentication tag $y^{\prime}$ for a value $s^{\prime} \neq s$ such that $\left(s^{\prime}, y^{\prime}, z\right.$, $\left.\alpha_{i, k}\right)$ is 1-consistent is equivalent to finding $\alpha_{i, k}$.

Proof. If both $\left(s, y, z, \alpha_{i, k}\right)$ and $\left(s^{\prime}, y^{\prime}, z, \alpha_{i, k}\right)$ are 1-consistent, then also $\left(s-s^{\prime}, y-\right.$ $\left.y^{\prime}, 0, \alpha_{i, k}\right)$ is 1 -consistent. The corresponding polynomial of degree 1 is not parallel to the $x$-axis, as $s-s^{\prime} \neq 0$. Thus it has an unique root at $\alpha_{i, k}=\frac{s-s^{\prime}}{s-s^{\prime}-y+y^{\prime}}$.

Lemma 9. The players $P_{j}$ and $P_{k}$ can locally compute an authentication and a verification tag of any linear combination of $\left(P_{i}, P_{j}, P_{k}\right)$-authenticated values (for fixed $\left.P_{i}\right)$. This is called the linearity of the authentication.

Proof. Let $s_{a}$ and $s_{b}$ be $\left(P_{i}, P_{j}, P_{k}\right)$-authenticated with authentication tags $y_{a}, y_{b}$ and verification tags $z_{a}, z_{b}$ and the (fixed) point $\alpha_{i, k}$ and let $L$ be a linear function. Then $L\left(s_{a}, s_{b}\right)$ is $\left(P_{i}, P_{j}, P_{k}\right)$-authenticated with authentication tag $y=L\left(y_{a}, y_{b}\right)$ and verification tag $z=L\left(z_{a}, z_{b}\right)$. This works as the polynomials of degree 1 over $\mathbb{F}$ form a vector space, hence $\left(L\left(s_{a}, s_{b}\right), L\left(y_{a}, y_{b}\right), L\left(z_{a}, z_{b}\right), \alpha_{i, k}\right)$ is 1-consistent.

Let $s$ be a value known to $P_{j}$ and $P_{k}$. Then these players can use the protocol Default Authenticate to $\left(P_{i}, P_{j}, P_{k}\right)$-authenticate $s$ without communication for arbitrary $P_{i}$. Note that $P_{i}$ does not play an (active) role in this protocol.

Protocol DefaultAuthenticate $\left(\boldsymbol{P}_{\boldsymbol{i}}, \boldsymbol{P}_{\boldsymbol{j}}, \boldsymbol{P}_{\boldsymbol{k}}, \boldsymbol{s}\right)$

0: $P_{j}, P_{k}$ take the value $s$ as input.

1: $P_{j}$ outputs authentication tag $y=s . P_{k}$ outputs verification $\operatorname{tag} z=s$. 
Lemma 10. If the value $s$ is known to the honest players in $\left\{P_{j}, P_{k}\right\}$ protocol Default Authenticate $\left(P_{i}, P_{j}, P_{k}, s\right)$ securely $\left(P_{i}, P_{j}, P_{k}\right)$-authenticates $s$ without any communication.

Proof. Correctness: $\left(s, s, s, \alpha_{i, k}\right)$ is 1-consistent for any $\alpha_{i, k}$. Privacy/Communication: No communication occurs.

The non-robust protocol Authenticate allows to securely $\left(P_{i}, P_{j}, P_{k}\right)$-authenticate a (secret) value $s$.

\section{Protocol Authenticate $\left(\boldsymbol{P}_{\boldsymbol{i}}, \boldsymbol{P}_{\boldsymbol{j}}, \boldsymbol{P}_{\boldsymbol{k}}, \boldsymbol{s}\right)$}

0: $P_{i}$ and $P_{j}$ take the value $s$ as input.

1: $P_{i}$ chooses random values $(y, z) \in \mathbb{F}$ such that $\left(s, y, z, \alpha_{i, k}\right)$ is 1 -consistent and random values $\left(s^{\prime}, y^{\prime}, z^{\prime}\right) \in \mathbb{F}$ such that $\left(s^{\prime}, y^{\prime}, z^{\prime}, \alpha_{i, k}\right)$ is 1 -consistent and sends $\left(s^{\prime}, y, y^{\prime}\right)$ to $P_{j}$ and $\left(z, z^{\prime}\right)$ to $P_{k}$

2: $P_{k}$ broadcasts random $r \in \mathbb{F}$.

3: $P_{i}$ broadcasts $s^{\prime \prime}=r s+s^{\prime}$ and $y^{\prime \prime}=r y+y^{\prime}$.

4: $P_{j}$ checks if $s^{\prime \prime}=r s+s^{\prime}$ and $y^{\prime \prime}=r y+y^{\prime}$ and broadcast OK or NOK accordingly. If NOK was broadcast the protocol is aborted.

5: $P_{k}$ checks if $\left(s^{\prime \prime}, y^{\prime \prime}, r z+z^{\prime}, \alpha_{i, k}\right)$ is 1 -consistent. If yes $P_{k}$ sends OK to $P_{j}$ otherwise he sends $\left(\alpha_{i, k}, z\right)$ to $P_{j}$, who adjusts $y$ such that $\left(s, y, z, \alpha_{i, k}\right)$ is 1-consistent.

6: $P_{j}$ outputs $y$ and $P_{k}$ outputs $z$.

Lemma 11. If $P_{k}$ is honest and $s$ is known to the honest players in $\left\{P_{i}, P_{j}\right\}$. Then Authenticate $\left(P_{i}, P_{j}, P_{k}, s\right)$ either securely $\left(P_{i}, P_{j}, P_{k}\right)$-authenticates $s$ or aborts except with error probability of at most $\frac{1}{|\mathbb{F}|}$. In the case of an abort a player in $\left\{P_{i}, P_{j}\right\}$ is corrupted. The protocol communicates at most $7 \log |\mathbb{F}|$ bits and broadcasts at most $3 \log |\mathbb{F}|+1$ bits.

Proof. Correctness: If the protocol was aborted, either $s^{\prime \prime} \neq r s+s^{\prime}$ or $y^{\prime \prime} \neq r y+y^{\prime}$ meaning $P_{i}$ is corrupted, or $P_{j}$ misleadingly accused $P_{i}$. Otherwise, the players use some $\left(s, y, z, \alpha_{i, k}\right)$ as authentication of $s$. The probability that $\left(s, y, z, \alpha_{i, k}\right)$ is not 1-consistent is $|\mathbb{F}|^{-1}$, as for a fixed $r$ there is exactly one way to choose $y, z$ such that the inconsistency is not detected. Privacy: The verification tag $z$, the values $s^{\prime \prime}$ and $y^{\prime \prime}$ are statistically independent of the value $s$. Also $\alpha_{i, k}$ is sent only to $P_{j}$ if either $P_{i}$ or $P_{k}$ is malicious. Communication: Seen by counting the number of messages sent or broadcast during the protocol.

Remark 1. If the (honest) players $P_{i}$ and $P_{j}$ do not know the same $s$ the protocol will abort as well.

Assume that $P_{k}$ knows a candidate $s^{\prime}$ for a $\left(P_{i}, P_{j}, P_{k}\right)$-authenticated value $s$. If $P_{j}$ wants to prove the authenticity of $s^{\prime}$ (i.e. that $s^{\prime}=s$ ) the players invoke the protocol Verify. If $P_{k}$ accepts the proof he outputs $s^{\prime}$, otherwise he outputs $\perp$. 
$\operatorname{Protocol}$ Verify $\left(\boldsymbol{P}_{\boldsymbol{i}}, \boldsymbol{P}_{j}, \boldsymbol{P}_{\boldsymbol{k}}, \boldsymbol{s}^{\prime}, \boldsymbol{A}_{\boldsymbol{i}, \boldsymbol{j}, \boldsymbol{k}}(s)\right)$

0: Let $A_{i, j, k}(s)=\left(y, z, \alpha_{i, k}\right)$. $P_{j}$ takes $y$ as input and $P_{k}$ takes $s^{\prime}, z$ as input.

1: $P_{j}$ sends $y$ to $P_{k}$

2: $P_{k}$ outputs $s^{\prime}$ if $\left(s^{\prime}, y, z, \alpha_{i, k}\right)$ is 1 -consistent otherwise $\perp$.

Lemma 12. Assume $s$ is $\left(P_{i}, P_{j}, P_{k}\right)$-authenticated and let $P_{k}$ be an honest player knowing $s^{\prime}$. If $P_{j}$ is honest and $s^{\prime}=s, P_{k}$ will output $s$ in Verify. Otherwise $P_{k}$ will output $\perp$ or s except with error probability of at most $\frac{1}{|\mathbb{|}|-2}$. The protocol communicates at most $\log |\mathbb{F}|$ bits.

Proof. Correctness: Let $P_{k}$ be an honest player,let $A_{i, j, k}(s)=\left(y, z, \alpha_{i, k}\right)$ be consistent with $s$, i.e. $\left(s, y, z, \alpha_{i, k}\right)$ is 1 -consistent and assume that $s^{\prime}=s$. If $P_{j}$ sends the right $y$ the vector $\left(s^{\prime}, y, z, \alpha_{i, k}\right)$ is 1-consistent and $P_{k}$ will output $s$. Otherwise $P_{k}$ always outputs $\perp$. So assume $s^{\prime} \neq s$. Then the probability of finding $y^{\prime}$ such that the vector $\left(s^{\prime}, y^{\prime}, z, \alpha_{i, k}\right)$ is 1 -consistent is at most $\frac{1}{|F|-2}$, thus $P_{k}$ outputs $\perp$ except with error probability of at most $\frac{1}{|\mathbb{F}|-2}$. Privacy/Communication: No information except $y$ is sent.

\subsection{Unconditional Secret Sharing}

Starting from the secret sharing of Section 3.1 we construct a sharing scheme for the $\mathcal{Q}^{2}$ case using the information-checking scheme of the previous section.

Definition 3. A value $s$ is shared with respect to the sharing specification $\mathbb{S}=\left(S_{1}, \ldots\right.$, $S_{h}$ ), if the following holds:

a) There exist shares $s_{1}, \ldots, s_{h}$ such that $s=\sum_{q=1}^{h} s_{q}$

c) $\forall P_{i}, P_{j} \in S_{q} P_{k} \in \mathcal{P} s_{q}$ is $\left(P_{i}, P_{j}, P_{k}\right)$-authenticated.

We denote the sharing of a value $s$ by $[s]$. Let $[s]_{q}=\left(s_{q},\left\{A_{i, j, k}\left(s_{q}\right)\right\}\right)$, where $s_{q}$ is the $q$-th share and $\left\{A_{i, j, k}\left(s_{q}\right)\right\}$ the set of all associated authentications. As the perfect sharing from Section 3.1 this sharing is linear and does not leak information to the adversary (for a $\mathcal{Z}$-private $\mathbb{S}$ ).

The following protocol allows a dealer $P_{D}$ to securely share a secret value $s$.

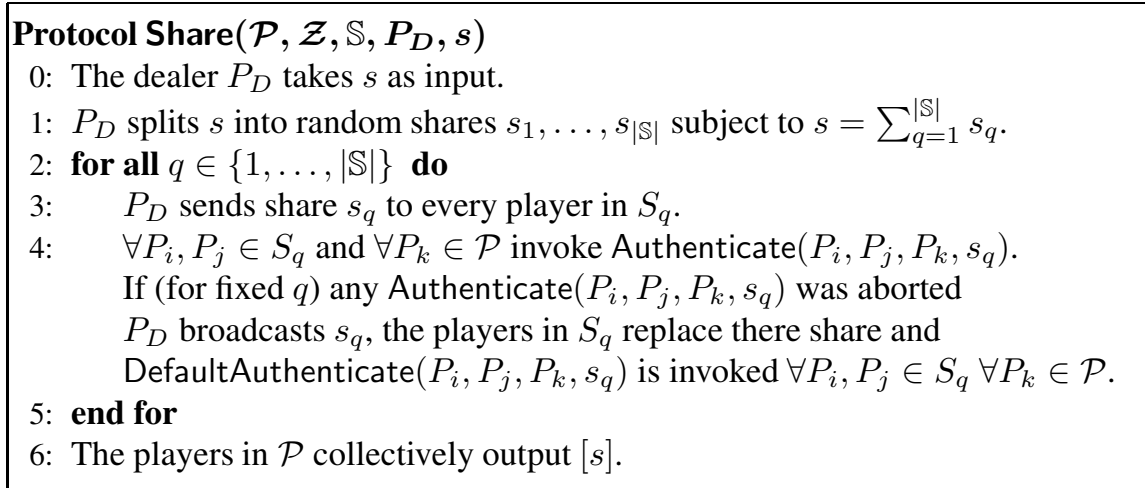


Lemma 13. For any adversary structure $\mathcal{Z}$ the protocol $\operatorname{Share}\left(\mathcal{P}, \mathcal{Z}, \mathbb{S}, P_{D}, s\right)$ securely computes a sharing $\left[s^{\prime}\right]$ except with error probability of at most $\frac{1}{|\mathbb{F}|} n^{3}|\mathbb{S}|$ and if $P_{D}$ is honest $s^{\prime}=s$. The protocol communicates at most $|\mathbb{S}|\left(7 n^{3}+n\right) \log |\mathbb{F}|$ bits and broadcasts at most $|\mathbb{S}|\left(\left(3 n^{3}+1\right) \log |\mathbb{F}|+n^{3}\right)$ bits.

Proof. Correctness: Assume that $P_{D}$ does not send the same value to the (honest) players in $S_{q}$ (Step 3). In this case at least one invocation of Authenticate will abort (see Remark 1) and $P_{D}$ must broadcast the value. Otherwise all (honest) player use the same value $s_{q}$ in Step 3. We have to show that every (honest) $P_{j}$ gets his authentications $A_{i, j, k}\left(s_{q}\right)$. If all instances of Authenticate do not abort the statement follows from Lemma 11 Otherwise $s_{q}$ is broadcast and the players use DefaultAuthenticate resulting in the proper sharing state (c.f. Lemma 10). Note that a single invocation of Authenticate has an error probability of at most $\frac{1}{|\mathbb{F}|}$, so the above upper bound on the error probability follows. Privacy: We only have to check that broadcasting $s_{q}$ in Step 4 does not violate privacy. But $s_{q}$ is only broadcast when at least one Authenticate was aborted. In this case either $P_{D}$ or a player in $S_{q}$ is malicious, hence $s_{q}$ is known to the adversary before the broadcast (Lemma 11 and Remark 1). Communication: Follows directly by counting the numbers of messages sent or broadcast (c.f. Lemmas 11 and 10,

If a value is publicly known the player can use DefaultShare to obtain a sharing of it.

\section{Protocol DefaultShare $(\mathcal{P}, \mathcal{Z}, \mathbb{S}, s)$}

0 : Every player takes $s$ as input.

1: The share $s_{1}$ is set to $s$ and all other shares are set to 0 .

2: DefaultAuthenticate $\left(P_{i}, P_{j}, P_{k}, s_{q}\right)$ is invoked $\forall S_{q} \forall P_{i}, P_{j} \in S_{q} \forall P_{k} \in \mathcal{P}$.

3: The players in $\mathcal{P}$ collectively output $[s]$.

Lemma 14. DefaultShare $(\mathcal{P}, \mathcal{Z}, \mathbb{S}, s)$ securely computes a sharing $[s]$ of $s$. The protocol does not communicate.

Proof. The statement follows from Lemmas 2 and 10.

The protocol ReconstructShare allows reconstruction of a share from some sharing $[s]$ to players in $R \subseteq \mathcal{P}$. Hence the players can reconstruct $s$ by invoking protocol ReconstructShare for each share of $[s]$.

Protocol ReconstructShare $\left(\mathcal{P}, \mathcal{Z}, \mathbb{S},[s]_{q}, R\right)$

0: The players in $S_{q}$ take collectively $[s]_{q}=\left(s_{q},\left\{A_{i, j, k}\left(s_{q}\right)\right\}\right)$ as input.

1: Every player $P_{j}$ in $S_{q}$ sends $s_{q}$ to every player in $R$.

2: for all $P_{j} \in S_{q}, P_{k} \in R$ do

3: $\quad$ Invoke $\operatorname{Verify}\left(P_{i}, P_{j}, P_{k}, s_{q}^{(j)}, A_{i, j, k}\left(s_{q}\right)\right) \forall P_{i} \in S_{q}$ where $s_{q}^{(j)}$ is the value received by $P_{k}$ from $P_{j}$ in Step1 If $P_{k}$ output $s_{q}^{(j)}$ in each invocation he accepts it as value for $s_{q}$.

\section{4: end for}

5: Each $P_{k}$ outputs some value he accepted in Step 3 (or $\perp$ if never accepted a value). 
Lemma 15. Assume $S_{q}$ and $\mathcal{Z}$ satisfy $\mathcal{Q}^{1}\left(S_{q}, \mathcal{Z}\right)$ and let $[s]_{q}$ be a consistent share. Every honest player in $R$ outputs $s_{q}$ in ReconstructShare except with error probability of at most $\frac{1}{|\mathbb{F}|-2} n\left|S_{q}\right|$. The protocol communicates at most $\left(n^{3}+n^{2}\right) \log |\mathbb{F}|$ bits and does not broadcast.

Proof. Correctness: As $S_{q}$ and $\mathcal{Z}$ satisfy $\mathcal{Q}^{1}\left(S_{q}, \mathcal{Z}\right)$ there exists at least one honest player $P_{j}$ in $S_{q}$, who sends the right value $s_{q}$ to $P_{k} \in R$ in Step 1 . Hence every (honest) $P_{k}$ will accept $s_{q}$ in Step 3 from $P_{j}$, as $P_{j}$ has a valid authentication for $s_{q}$ from every player in $S_{q}$ (c.f. Lemma 12). On the other hand a malicious player does not have a valid authentication for $s_{q}^{\prime} \neq s_{q}$ from every player in $S_{q}$ (one of them is honest!). So no honest player will accept $s_{q}^{\prime} \neq s_{q}$ in Step 3 and thus $P_{k}$ output $s_{q}$ in the last step except with error probability of at most $\frac{1}{|\mathbb{F}|-2}\left|S_{q}\right|$ (c.f. Lemma 12). As there are at most $n$ players in $R$ the overall error probability follows. Privacy: Follows from Lemma 12. Communication: Follows directly by counting the numbers of messages sent (c.f. Lemma 12]

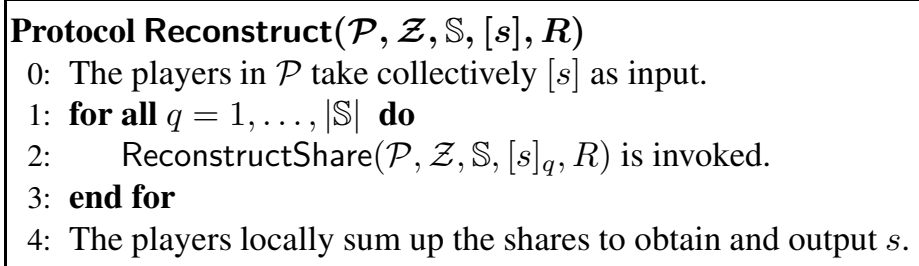

Lemma 16. Assume $\mathbb{S}$ and $\mathcal{Z}$ satisfy $\mathcal{Q}^{1}(\mathbb{S}, \mathcal{Z})$ and let $[s]$ be a sharing of the value $s$. Every honest player in $R$ outputs $s$ in Reconstruct except with error probability of at most $\frac{1}{|\mathbb{F}|-2} n^{2}|\mathbb{S}|$. The protocol communicates at most $|\mathbb{S}|\left(n^{3}+n^{2}\right) \log |\mathbb{F}|$ bits and does not broadcast.

Proof. The statement follows directly from Lemma 15, as the players invoke the protocol ReconstructShare for each share.

\subsection{Multiplication}

We present a protocol for the unconditionally-secure computation of the (shared) product of two shared values $[a]$ and $[b]$. The idea is, as in the perfect case, to use an optimistic multiplication. The protocol BasicMult takes a set $M$ of (identified) malicious players as input and outputs the correct product given that no player in $\mathcal{P} \backslash M$ actively cheated. In a next step a probabilistic check is used to determine whether the product computed in BasicMult is correct. This allows us to detect malicious behaviour. If cheating occured, all involved sharings (from BasicMult) are reconstructed to identify a cheater in $\mathcal{P} \backslash M$. These reconstructions violate the privacy of the involved factors the protocol is not used directly in the actual circuit computation. Instead we use it to multiply two random values and make use of circuit randomization from [Bea91b] for actual multiplication gates. 
Protocol BasicMult $(\mathcal{P}, \mathcal{Z}, \mathbb{S},[a],[b], M)$

0 : The players in $\mathcal{P}$ take collectively $[a],[b]$ and $M$ as input.

1: $\forall S_{q}: S_{q} \cap M \neq \emptyset$ invoke ReconstructShare to reconstruct $a_{q}$ and $b_{q}$.

2: a) Each player $P_{i} \in \mathcal{P} \backslash M$ (locally) computes his designated products and shares the sum $c_{i}=\sum_{(p, q) \in I(i)} a_{p} b_{q}$.

b) For each $P_{i} \in M$ DefaultShare $\left(\mathcal{P}, \mathcal{Z}, \mathbb{S}, c_{i}\right)$ is invoked where $c_{i}=$ $\sum_{(p, q) \in I(i)} a_{p} b_{q}$.

3: The players collectively output $\left(\left[c_{1}\right], \ldots,\left[c_{n}\right]\right)$ and $[c]=\sum_{i=1}^{n}\left[c_{i}\right]$.

Lemma 17. Let $M \subseteq Z^{*}$ be a set of (identified) malicious players and assume that $\mathcal{Z}$ and $\mathbb{S}$ satisfy $\mathcal{Q}^{1}(\mathbb{S}, \mathcal{Z})$. Then BasicMult $(\mathcal{P}, \mathcal{Z}, \mathbb{S},[a],[b], M)$ securely computes sharings $[c],\left(\left[c_{1}\right], \ldots,\left[c_{n}\right]\right)$ except with error probability of $\mathcal{O}\left(\frac{1}{|\mathbb{F}|} n^{4}|\mathbb{S}|\right)$. If no player in $\mathcal{P} \backslash M$ actively cheats, then $\forall i c_{i}=\sum_{(p, q) \in I_{Z}(i)} a_{p} b_{q}$ and $c=a b$. The protocol communicates at most $\mathcal{O}\left(|\mathbb{S}| n^{4} \log |\mathbb{F}|\right)$ bits and broadcasts at most $\mathcal{O}\left(|\mathbb{S}| n^{4} \log |\mathbb{F}|\right)$ bits.

Proof. Correctness: The properties of the sharing protocol guarantee that the outputs are valid sharings except with error probability of $\mathcal{O}\left(\frac{1}{|\mathbb{F}|} n^{4}|\mathbb{S}|\right)$. The $\mathcal{Q}^{1}(\mathbb{S}, \mathcal{Z})$ property allows the players to securely reconstruct shares and grants that there exists a proper assignment of players in $\mathcal{P}$ to the local products. If none of the players in $\mathcal{P} \backslash M$ cheated, it holds for each $P_{i}$ that $c_{i}=\sum_{(p, q) \in I_{Z}(i)} a_{p} b_{q}$ (for players in $M$ DefaultShare is used on reconstructed values). Privacy: All reconstructed shares $a_{q}, b_{q}$ are known to players in $M$. Complexity: Follow directly from the properties of the sharing scheme (c.f. Lemmas 13, 14 and 15).

Detectable Random Triple Generation. The following unconditionally secure protocol takes a set $M$ of malicious players as an additional input and computes a random multiplication triple $([a],[b],[c])$ where $c=a b$ given that no player in $\mathcal{P} \backslash M$ actively cheats. Otherwise it outputs a set of malicious players $M^{\prime}$ such that $M \subsetneq M^{\prime}$. This protocol uses a probabilistic check to detect cheating. First the players generate a shared random challenge $[r]$ and a blinding $\left[b^{\prime}\right]$. Then they use BasicMult to compute the sharings $[c]=[a][b],\left[c^{\prime}\right]=[a]\left[b^{\prime}\right]$ and check whether $[a]\left(r[b]+\left[b^{\prime}\right]\right)=\left(r[c]+\left[c^{\prime}\right]\right)$. If this is the case the multiplication triple $([a],[b],[c])$ is output. Otherwise the players identify (at least) one cheater in $\mathcal{P} \backslash M$ by reconstructing $[a],[b],\left[b^{\prime}\right],[c],\left[c^{\prime}\right]$.

Lemma 18. If $\mathbb{S}$ and $\mathcal{Z}$ satisfy $\mathcal{Q}^{1}(\mathbb{S}, \mathcal{Z})$ and $M \subseteq Z^{*}$, the protocol RandomTriple outputs either a random multiplication triple $([a],[b],[c])$ or set $M^{\prime} \subseteq Z^{*}$ where $M \subsetneq$ $M^{\prime}$ except with error probability of $\mathcal{O}\left(\frac{1}{|\mathbb{F}|}|\mathbb{S}| n^{4}\right)+\frac{1}{|\mathbb{F}|}$. No information is leaked to the adversary. RandomTriple communicates at most $\mathcal{O}\left(|\mathbb{S}| n^{4} \log |\mathbb{F}|\right)$ bits and broadcasts at most $\mathcal{O}\left(|\mathbb{S}| n^{4} \log |\mathbb{F}|\right)$ bits.

Proof. Correctness: In Step 2, the players compute $[c]$ and $\left[c^{\prime}\right]$. Given that no player in $\mathcal{P} \backslash M$ actively cheated it holds that $c=a b$ and $c^{\prime}=a b^{\prime}$. In this case $[a]\left(r[b]+\left[b^{\prime}\right]\right)-$ $r[c]-\left[c^{\prime}\right]$, which is computed in Step3, is zero for all $r$ and the players reconstruct the random multiplication triple $([a],[b],[c])$. If $c \neq a b$ the difference $[a]\left(r[b]+\left[b^{\prime}\right]\right)-r[c]-$ 
$\left[c^{\prime}\right]$ is non-zero except for at most one $r$ and the players go to Step 5 with probability at least $\left(1-\frac{1}{|\mathbb{F}|}\right)$ (assuming that no errors happen in sharing and reconstruction of values). For at least one player $P_{i} \in \mathcal{P} \backslash M$ it must hold that $r c_{i}+c_{i}^{\prime} \neq \sum_{(p, q) \in I(i)} r\left(a_{p} b_{q}\right)+$ $\left(a_{p} b_{q}^{\prime}\right)$. By opening all involved sharing it is easy to find these players. Thus it holds that $M \subsetneq M^{\prime}$ and $M^{\prime} \subseteq Z^{*}$. The overall error probability is composed of the error probability of the sharing scheme and the one of the random challenge check in Step 3. Privacy: Neither the protocol BasicMult nor the sharing scheme do violate privacy (c.f. Lemma 17). The values $e$ is statistically independent of $([a],[b],[c])$, as $b^{\prime}$ acts as blinding. If no cheating occurred the value $d$ is always zero. If Step 5 is invoked, the reconstructed values are not used, and privacy is met. Communication: Follows from counting the number of messages sent (c.f. Lemmas 13, 16 and 17).

\section{Protocol Random $\operatorname{Triple}(\mathcal{P}, \mathcal{Z}, \mathbb{S}, M)$}

0 : The players take the set $M \subseteq \mathcal{P}$ as input.

1: The players generate random shared values $[a],[b],\left[b^{\prime}\right],[r]$ by summing up shared random values (one from each player) for each value.

2: Invoke BasicMult $(\mathcal{P}, \mathcal{Z}, \mathbb{S},[a],[b], M)$ to compute the sharing $[c]$ and the vector $\left(\left[c_{1}\right], \ldots,\left[c_{n}\right]\right)$ and invoke BasicMult $\left(\mathcal{P}, \mathcal{Z}, \mathbb{S},[a],\left[b^{\prime}\right], M\right)$ to compute the sharing $\left[c^{\prime}\right]$ and the vector $\left(\left[c_{1}^{\prime}\right], \ldots,\left[c_{n}^{\prime}\right]\right)$.

3: Reconstruct $[r]$ and (locally) compute $[e]=r[b]+\left[b^{\prime}\right]$ and reconstruct it to obtain $e$. Then $[d]=e[a]-r[c]-\left[c^{\prime}\right]$ is computed (locally) and reconstructed.

4: If the value $d$ is zero the players output $([a],[b],[c])$.

5: Otherwise reconstruct the sharings $[a],[b],\left[b^{\prime}\right],\left[c_{1}\right], \ldots,\left[c_{n}\right],\left[c_{1}^{\prime}\right], \ldots,\left[c_{n}^{\prime}\right]$. The players output $M^{\prime}=M \cup\left\{P_{i}: r c_{i}+c_{i}^{\prime} \neq \sum_{(p, q) \in I(i)} r\left(a_{p} b_{q}\right)+\left(a_{p} b_{q}^{\prime}\right)\right\}$.

Multiplication with Circuit Randomization. The actual multiplication is based on circuit randomization [Bea91b]. It allows players to compute the product $[x y]$ of two shared values $[x]$ and $[y]$ at the cost of two reconstructions given a random multiplication triple $([a],[b],[c])$, where $a b=c$. The trick is to use that $x y=((x-a)+a)((y-$ $b)+b)$. By reconstructing $d=x-a$ and $e=y-b$ the players can compute $[x y]$ as $d e+d[b]+[a] e+[c]$. This does not violate the secrecy of $[x]$ or $[y]$ as the random values $[a]$ and $[b]$ act as blinding.

\section{Protocol Multiplication $(\mathcal{P}, \mathcal{Z}, \mathbb{S},[x],[y])$}

0 : The players in $\mathcal{P}$ take collectively $[x],[y]$ as input and set $M:=\emptyset$.

1: Invoke Random $\operatorname{Triple}(\mathcal{P}, \mathcal{Z}, \mathbb{S}, M)$. If the protocol outputs a set $M^{\prime}$, set $M \leftarrow$ $M^{\prime}$ and repeat Step 1. Otherwise use the output as random multiplication triple $([a],[b],[c])$.

2: Compute and reconstruct $\left[d_{x}\right]=[x]-[a]$ and $\left[d_{y}\right]=[y]-[b]$. Compute $d_{x} d_{y}+d_{x}[b]+d_{y}[a]+[c]=[x y]$ to obtain a sharing of $x y$. 
Lemma 19. Multiplication $(\mathcal{P}, \mathcal{Z}, \mathbb{S},[x],[y])$ is an unconditional secure multiplication protocol given that $\mathbb{S}$ and $\mathcal{Z}$ satisfy $\mathcal{Q}^{1}(\mathbb{S}, \mathcal{Z})$. The protocol has an error probability of $\mathcal{O}\left(\frac{1}{|\mathbb{F}|}|\mathbb{S}| n^{5}\right)$, communicates at most $\mathcal{O}\left(|\mathbb{S}| n^{5} \log |\mathbb{F}|\right)$ bits and broadcasts at most $\mathcal{O}\left(|\mathbb{S}| n^{5} \log |\mathbb{F}|\right)$ bits.

Proof. Correctness: Assume that RandomTriple in Step 1 outputs a set $M^{\prime}$, then we have that $M \subsetneq M^{\prime} \subseteq \mathcal{P}$. Hence this step is repeated less then $n$ times and results in a random multiplication triple $([a],[b],[c])$ (c.f. Lemma 18). The rest of the protocol is just the multiplication from [Bea91b]. Privacy: Follows from [Bea91b] and Lemma 18. Communication: Follows from counting the number of messages sent (c.f. Lemmas 16 and 18).

\subsection{Unconditional MPC Protocol}

The combination of Share, Reconstruct and Multiplication directly gives the following unconditionally secure MPC protocol.

\section{$\operatorname{Protocol} \operatorname{MPC}(\mathcal{P}, \mathcal{Z}, \mathcal{C})$}

0 : The players take $\mathbb{S}:=\{\mathcal{P} \backslash Z \mid Z \in \mathcal{Z}\}$ as sharing specification.

1: For every gate of $\mathcal{C}$ being evaluated do the following:

- Input gate for $P_{D}$ : $\operatorname{Share}\left(\mathcal{P}, \mathcal{Z}, \mathbb{S}, P_{D}, s\right)$ is invoked to share $s$

- Linear gate: The linear combination of the corresponding shares is computed locally using the linearity of the sharing.

- Random gate: Each player shares a random value. The sum of these values is used as output of the gate.

- Multiplication gate: Multiplication $(\mathcal{P}, \mathcal{Z}, \mathbb{S},[x],[y])$ is used to multiply $[x]$ and $[y]$.

- Output gate: The players invoke $\operatorname{Reconstruct}(\mathcal{P}, \mathcal{Z}, \mathbb{S},[s], R)$ to reconstruct $s$ for players in $R$.

Theorem 2. Let $\mathcal{C}$ be a circuit over $\mathbb{F}$, where $|F| \in \Omega\left(2^{\kappa}\right)$ and $\kappa$ is a security parameter, and let $\mathcal{Z}$ be an adversary structure satisfying $\mathcal{Q}^{2}(\mathcal{P}, \mathcal{Z})$, then $\operatorname{MPC}(\mathcal{P}, \mathcal{Z}, \mathcal{C})$ $\mathcal{Z}$-securely evaluates $\mathcal{C}$ with an error probability of $2^{-\kappa}|\mathcal{C}||\mathcal{Z}| \cdot \operatorname{Poly}(n, \kappa)$. It communicates $|\mathcal{C}||\mathcal{Z}| \cdot \operatorname{Poly}(n, \kappa)$ bits and broadcasts $|\mathcal{C}||\mathcal{Z}| \cdot \operatorname{Poly}(n, \kappa)$ bits within $\operatorname{Poly}(n, \kappa) \cdot d$ rounds, where d denotes the multiplicative depth of $C$.

Proof. It is easy to see that $\mathbb{S}:=\{\mathcal{P} \backslash Z \mid Z \in \mathcal{Z}\}$ is a sharing specification satisfying $\mathcal{Q}^{1}(\mathbb{S}, \mathcal{Z})$. Hence by the properties of the sharing scheme and Lemma 19 correctness and the bound on the error probability follow. The claimed communication and broadcast complexity follow directly from the used subprotocols. Inspection of the subprotocols also shows that it is possible to evaluate gates on the same multiplicative depth of $C$ in parallel. As each subprotocol only requires $\operatorname{Poly}(n, \kappa)$ rounds, the total number of rounds follows. 
Note that broadcast can be (unconditionally secure) simulated using the protocol from [PW96], which communicates $\operatorname{Poly}(n, \kappa)$ bits in order to broadcast one bit (with error probability of $\mathcal{O}\left(2^{-\kappa}\right)$ ). This results in an MPC protocol with the same efficiency and error probability as stated in Theorem 2 .

The error probability of the presented protocol grows linearly in the size of the adversary structure $\mathcal{Z}$. As $|\mathcal{Z}|$ is typically exponential in $n$, the security parameter $\kappa$ must be chosen accordingly (such that $|\mathcal{Z}| \in \operatorname{Poly}(\kappa)$ ). This results in a huge security parameter and therefore in inefficient protocols. We therefore provide an extension of the previous protocol in which the error probability only depends on $\log |Z|$, and hence a reasonably large security parameter $\kappa$ is sufficient.

\section{Unconditional Protocol for Superpolynomial $|\mathcal{Z}|$}

The protocol from the previous section has an error probability linear in $|\mathcal{Z}|$, which is problematic for large adversary structures $\mathcal{Z}$. In this section, we present modifications to the protocol that reduce the dependency to $\log |\mathcal{Z}|$, which is in $\operatorname{Poly}(n)$.

The reason for the error probability being dependent on $|\mathcal{Z}|$ is twofold: Firstly, the protocol requires $\Omega(|\mathcal{Z}|)$ probabilistic checks, in each of them a cheating party might remain undetected with probability $2^{-\kappa}$. Secondly, the protocol requires $\Omega(|\mathcal{Z}|)$ broadcasts, each of them having a small probability of failure.

\subsection{Information Checking}

In each invocation of Authenticate / Verify, a cheating attempt of a malicious player $P_{i}$ is not detected with probability of $\mathcal{O}\left(\frac{1}{|\mathbb{F}|}\right)$ (c.f. Section 4.1). As these protocols are invoked $\Theta(|\mathcal{Z}|)$ times per sharing, the resulting error probability depends linearly on $|\mathcal{Z}|$. To avoid this we use local dispute control to deal with detected cheaters.

More formally, each player $P_{k}$ locally maintains a list $\mathcal{L}_{k}$ of players whom he distrusts. At the beginning of the MPC protocol these lists are empty. Protocol Authenticate is modified, such that $P_{j}$ puts $P_{i}$ on his list $\mathcal{L}_{j}$ if the check in Step 4 fails. Once $P_{i} \in \mathcal{L}_{j}, P_{j}$ behaves in all future invocations of the protocol as if the check in Step 4 failed independently whether this is the case or not. Similarly $P_{k}$ puts $P_{i}$ on his list $\mathcal{L}_{k}$ if the check in Step 5 fails. As soon as $P_{i} \in \mathcal{L}_{k}, P_{k}$ behaves in Step 5 as if the corresponding check failed. Furthermore, in protocol Verify, $P_{k}$ puts $P_{j}$ on his list $\mathcal{L}_{k}$ if the check in Step2 failed. Again $P_{k}$ behaves for all $P_{j} \in \mathcal{L}_{k}$ as if the check failed independently whether this is the case or not.

In both protocols the adversary has a chance of $\mathcal{O}\left(\frac{1}{|\mathbb{F}|}\right)$ to cheat successfully, but if he fails (with probability $\Omega\left(1-\frac{1}{|\mathbb{F}|}\right)$ ) one corrupted player $P_{i}$ is put on the list $\mathcal{L}_{k}$ of an honest player $P_{k}$. From then on $P_{i}$ is never able to cheat in instances of both protocols when $P_{k}$ takes part (in the right position). This means that the adversary actually has at most $n^{2}$ attempts to cheat. Hence total error probability of arbitrary many instances of Verify and Authenticate is at most $\mathcal{O}\left(\frac{1}{|\mathbb{F}|} n^{2}\right)$ and no longer depends on $\mathcal{Z}$.

Note that the parallel invocation of Authenticate, as it is used in Share, requires special care. For example if in one of the parallel invocations of Authenticate (with $P_{i}$ 
and $P_{k}$ ) the consistency check fails $P_{k}$ must assume that all other parallel checks failed. Analogous modifications are made in Verify and Multiplication.

Lemma 20. The modified Authenticate and Verify protocols have a total error probability of $\mathcal{O}\left(\frac{1}{|\mathbb{F}|} n^{2}\right)$ independent of the number of invocations.

\subsection{Broadcast}

Although broadcast is only needed in Share, the total number of broadcast calls is in $\Theta(|\mathcal{Z}|)$. If [PW96] is used, the resulting overall error probability depends linearly on $|\mathcal{Z}|$. To avoid this problem, the number of broadcast calls must be reduced.

To reach this goal we use the fact that the Share protocol only has constantly many rounds. In each round a player $P_{S}$ must broadcast $\Theta(|\mathcal{Z}|)$ many messages of size $\mathcal{O}(\log |\mathbb{F}|)$. Instead of broadcasting these messages in parallel, $P_{S}$ sends their concatenation to the other players, who then check that they received the same message. If an inconsistency is detected the protocol is repeated. To limit the number of repetitions we use the concept of dispute control from [BH06] which prevents the malicious players from repetitive cheating. Dispute control is realized by a publicly known dispute set $\Gamma \subseteq \mathcal{P} \times \mathcal{P}$, a set of unordered pairs of players. If $\left\{P_{i}, P_{j}\right\} \in \Gamma$ it means that there is a dispute between $P_{i}$ and $P_{j}$ and thus at least one of them is corrupted. Note that from $P_{i}$ 's view all player in $\left\{P_{j} \mid\left\{P_{i}, P_{j}\right\} \in \Gamma\right\}$ are malicious and thus he no longer trust them. At the beginning of the MPC protocol $\Gamma$ is empty.

\section{Protocol OptimisticBroadcast $\left(\mathcal{P}, \mathcal{Z}, P_{S}, \boldsymbol{m}\right)$}

0: The player $P_{S}$ takes $m \in\{0,1\}^{w}$ as input.

1: $\forall\left\{P_{i}, P_{S}\right\} \notin \Gamma P_{S}$ sends $m$ as $m_{i}$ to $P_{i}$.

2: $\forall\left\{P_{i}, P_{j}\right\} \notin \Gamma P_{i}$ sends $m_{i}$ as $m_{i j}$ to $P_{j}$.

3: $\forall P_{i}$ if all received values are the same $P_{i}$ is happy, otherwise unhappy. $P_{i}$ broadcasts using [PW96] his happy bit.

4: If all players are happy, each $P_{i}$ outputs the value he holds.

Otherwise, an unhappy player $P_{i}$ (e.g. the one with the smallest index) broadcasts $j, j^{\prime}, z, b$ where $m_{j i}$ differs from $m_{j^{\prime} i}$ at bit-position $z$ and $b$ is the bit of $m_{j i}$ at position $z$. Then $P_{S}, P_{j}, P_{j^{\prime}}$ broadcast their versions of the bit at position $z$. Using this information the players localize a dispute between two players of $\left\{P_{i}, P_{S}, P_{j}, P_{j^{\prime}}\right\}$. Then the protocol is repeated with updated $\Gamma$.

Lemma 21. The protocol OptimisticBroadcast $\left(\mathcal{P}, \mathcal{Z}, P_{S}, m\right)$ achieves the broadcast of a message $m^{\prime} \in\{0,1\}^{w}$. The protocol communicates at most $w \cdot \operatorname{Poly}(n, \kappa)$ bits and broadcasts at most $\log w \cdot \operatorname{Poly}(n, \kappa)$.

Proof. The properties of $\Gamma$ guarantee that honest players will exchange in Step 2 their received messages from $P_{S}$. So if all honest player are happy they all will output the same message $m^{\prime}$. For an honest $P_{S}$ this also ensures that $m^{\prime}=m$. If a player is unhappy, at least one player misbehaved. The actions taken in Step 4 then ensure that 
the honest players will find at least one dispute. The protocol will terminate, as the number of repetition is limited by $n(n-1)$. As the broadcast of $z$ requires $\log w$ bits, the communication and broadcast complexities follow.

For a message of length $\Theta(|\mathcal{Z}|)$ the above protocol only needs to broadcast $\log |\mathcal{Z}|$. $\operatorname{Poly}(n, \kappa)$ bits, hence the total number of broadcast calls per invocation of Share is reduced to $\log |\mathcal{Z}| \cdot \operatorname{Poly}(n, \kappa)$.

Lemma 22. The modified Share protocol communicates $|\mathcal{C}||\mathcal{Z}| \cdot \operatorname{Poly}(n, \kappa)$ bits and broadcasts $|\mathcal{C}| \log |\mathcal{Z}| \cdot \operatorname{Poly}(n, \kappa)$ bits.

\subsection{Summary}

The combination of the above extension results in the following Lemma:

Lemma 23. Let $\mathcal{C}$ be a circuit over $\mathbb{F}$, where $|\mathbb{F}| \in \Omega\left(2^{\kappa}\right)$ and $\kappa$ is a security parameter, and let $\mathcal{Z}$ be an adversary structure satisfying $\mathcal{Q}^{2}(\mathcal{P}, \mathcal{Z})$, then the modified protocol $\operatorname{MPC}(\mathcal{P}, \mathcal{Z}, \mathcal{C}) \mathcal{Z}$-securely evaluates $\mathcal{C}$ with an error probability of $2^{-\kappa}|\mathcal{C}| \cdot \operatorname{Poly}(n, \kappa)$. It communicates $|\mathcal{C}||\mathcal{Z}| \cdot \operatorname{Poly}(n, \kappa)$ bits and broadcasts $|\mathcal{C}| \log |\mathcal{Z}| \cdot \operatorname{Poly}(n, \kappa)$ bits. The number of rounds is $\operatorname{Poly}(n, \kappa) \cdot d$, where d denotes the multiplicative depth of $C$.

Proof. Follows directly from Theorem 2 and Lemmas 20 and 22.

By replacing broadcast with the simulated one from [PW96], one gets for $|\mathcal{Z}| \in \mathcal{O}\left(2^{n}\right)$ and $|\mathcal{C}| \in \operatorname{Poly}(\kappa)$ the following theorem.

Theorem 3. Let $\mathcal{C}$ be a circuit over $\mathbb{F}$, where $|\mathbb{F}| \in \Omega\left(2^{\kappa}\right)$ and $\kappa$ is a security parameter, and let $\mathcal{Z}$ be an adversary structure satisfying $\mathcal{Q}^{2}(\mathcal{P}, \mathcal{Z})$, then $\operatorname{MPC}(\mathcal{P}, \mathcal{Z}, \mathcal{C})$ $\mathcal{Z}$-securely evaluates $\mathcal{C}$ with an error probability of $2^{-\kappa} \cdot \operatorname{Poly}(n, \kappa)$. It communicates $|\mathcal{Z}| \cdot \operatorname{Poly}(n, \kappa)$ bits.

\section{Lower Bound on the Efficiency}

The following theorem states that there exists a family of circuits and $\mathcal{Q}^{2}$ adversary structures such that the length of unconditionally secure protocols tolerating these adversaries grows exponentially in the number of players. This implies that the computational complexity of our protocol from the previous section is optimal, as there exists no protocol with a computational complexity in $\mathrm{o}(|\mathcal{Z}|)$.

Theorem 4. [Hir01] Let $\mathcal{C}$ be the circuit which takes inputs from $P_{1}$ and $P_{2}$ and outputs the product to $P_{1}$. Then there exists a family $\mathcal{Z}_{2}, \mathcal{Z}_{3}, \ldots$ of $\mathcal{Q}^{2}$ adversary structures for player sets $\mathcal{P}_{2}, \mathcal{P}_{3}, \ldots\left(\left|\mathcal{P}_{n}\right|=n\right)$ such that the length of the shortest unconditionally $\mathcal{Z}_{n}$-secure protocol for $\mathcal{C}$ grows exponentially in $n$. 


\section{References}

[Bea91a] Beaver, D.: Secure multiparty protocols and zero-knowledge proof systems tolerating a faulty minority. Journal of Cryptology 4(2), 75-122 (1991)

[Bea91b] Beaver, D.: Efficient multiparty protocols using circuit randomization. In: Feigenbaum, J. (ed.) CRYPTO 1991. LNCS, vol. 576, pp. 420-432. Springer, Heidelberg (1992)

$\left[\mathrm{BFH}^{+} 08\right]$ Beerliová-Trubíniová, Z., Fitzi, M., Hirt, M., Maurer, U., Zikas, V.: MPC vs. SFE: Perfect security in a unified corruption model. In: Canetti, R. (ed.) TCC 2008. LNCS, vol. 4948, pp. 231-250. Springer, Heidelberg (2008)

[BGW88] Ben-Or, M., Goldwasser, S., Wigderson, A.: Completeness theorems for noncryptographic fault-tolerant distributed computation. In: STOC, pp. 1-10. ACM (1988)

[BH06] Beerliová-Trubíniová, Z., Hirt, M.: Efficient multi-party computation with dispute control. In: Halevi, S., Rabin, T. (eds.) TCC 2006. LNCS, vol. 3876, pp. 305-328. Springer, Heidelberg (2006)

[CCD88] Chaum, D., Crépeau, C., Damgard, I.: Multiparty unconditionally secure protocols. In: STOC, pp. 11-19. ACM (1988)

[CDD $\left.{ }^{+} 99\right]$ Cramer, R., Damgård, I.B., Dziembowski, S., Hirt, M., Rabin, T.: Efficient multiparty computations secure against an adaptive adversary. In: Stern, J. (ed.) EUROCRYPT 1999. LNCS, vol. 1592, p. 311. Springer, Heidelberg (1999)

[FM98] Fitzi, M., Maurer, U.: Efficient byzantine agreement secure against general adversaries. In: Kutten, S. (ed.) DISC 1998. LNCS, vol. 1499, pp. 134-148. Springer, Heidelberg (1998)

[GMW87] Goldwasser, S., Micali, S., Wigderson, A.: How to play any mental game, or a completeness theorem for protocols with an honest majority. In: STOC, vol. 87, pp. 218-229 (1987)

[Hir01] Hirt, M.: Multi-Party Computation: Efficient Protocols, General Adversaries, and Voting. PhD thesis, ETH Zurich (September 2001), Reprint as ETH Series in Information Security and Cryptography, vol. 3. Hartung-Gorre Verlag, Konstanz (2001) ISBN 3-89649-747-2

[HM97] Hirt, M., Maurer, U.: Complete characterization of adversaries tolerable in secure multi-party computation. In: PODC, pp. 25-34 (August 1997)

[HM00] Hirt, M., Maurer, U.: Player simulation and general adversary structures in perfect multiparty computation. Journal of Cryptology 13(1), 31-60 (2000), Extended abstract in Proc. 16th of ACM PODC 1997 (1997)

[HMZ08] Hirt, M., Maurer, U., Zikas, V.: MPC vs. SFE: Unconditional and computational security. In: Pieprzyk, J. (ed.) ASIACRYPT 2008. LNCS, vol. 5350, pp. 1-18. Springer, Heidelberg (2008)

[Mau02] Maurer, U.: Secure multi-party computation made simple. In: Cimato, S., Galdi, C., Persiano, G. (eds.) SCN 2002. LNCS, vol. 2576, pp. 14-28. Springer, Heidelberg (2003)

[PSR03] Prabhu, B., Srinathan, K., Pandu Rangan, C.: Trading players for efficiency in unconditional multiparty computation. In: Cimato, S., Galdi, C., Persiano, G. (eds.) SCN 2002. LNCS, vol. 2576, pp. 342-353. Springer, Heidelberg (2003)

[PW96] Pfitzmann, B., Waidner, M.: Information-theoretic pseudosignatures and byzantine agreement for $t \geq n / 3$. In Research report. IBM Research (1996)

[RB89] Rabin, T., Ben-Or, M.: Verifiable secret sharing and multiparty protocols with honest majority. In: STOC, pp. 73-85. ACM (1989)

[Yao82] Yao, A.C.: Protocols for secure computations. In: FOCS, pp. 160-164 (1982) 